\title{
Les bases de données TEPOQAL : pour une diffusion tangible de la poésie québécoise traduite en espagnol ${ }^{1}$
}

\author{
Madeleine Stratford (Université du Québec en Outaouais) \\ Elisa Calderón Contreras (Université du Québec en Outaouais) \\ et Marco Suárez (Université du Québec en Outaouais)
}

Depuis 1980, la poésie québécoise circule en Amérique latine, surtout au Mexique, en particulier depuis la participation du Québec à la Foire internationale du livre (FIL) de Guadalajara en 2003. En principe, il s'agit donc d'une littérature déjà diffusée en espagnol. Toutefois, les versions tendent à être difficiles d'accès : souvent tirées à peu d'exemplaires et épuisées, elles ne sont pas toujours répertoriées dans les catalogues de bibliothèques et dans ceux des maisons d'édition ou des distributeurs, et lorsqu'elles le sont, il n'est pas rare que les références soient incomplètes, voire erronées. En 2001, Graciela Martínez-Zalce déclare:

\footnotetext{
${ }^{1}$ Cette recherche a bénéficié du soutien du Fonds institutionnel de développement de la recherche de l'Université du Québec en Outaouais et du Fonds québécois de recherche en société et culture. Nous désirons aussi remercier Gabrielle-Émilie Audet pour sa contribution au projet à titre d'assistante de recherche.
} 
«There is still no market in Mexico for Canadian literature: it has not been publicized enough $^{2} »($ p. 70). Depuis, la situation ne semble pas avoir beaucoup évolué, car Luis Vicente de Aguinaga doute, en 2008, que sa traduction d'Hector de Saint-Denys Garneau reçoive beaucoup d'attention, le poète étant selon lui pratiquement inconnu au Mexique (cité dans Solis, 2008, par. 3). Ainsi, bien que la poésie québécoise soit de plus en plus traduite en espagnol et distribuée en Amérique latine, les versions jouissent souvent d'une diffusion mitigée (Stratford, 2013a et 2013b). Ce contraste n'est pas étonnant en soi, la poésie étant loin d'être un genre «vendeur». Cela dit, il y a lieu de s'étonner que la circulation des traductions ait été si faible quand on considère le battage médiatique qu'elles ont suscité autour de la FIL de 2003 (Stratford, 2013a) ainsi que la couverture élogieuse des poètes québécois dans la presse écrite mexicaine au cours de la première décennie du XXIe siècle (Stratford, 2013b).

Dans le but d'étudier le phénomène plus avant, nous avons entrepris en 2010, dans le cadre d'une recherche financée par le Fonds de recherche du Québec en Société et Culture (FRQ-SC), d'analyser les modes de traduction, de diffusion et de réception de la poésie québécoise dans les pays hispanophones d'Amérique latine, en particulier au Mexique et en Argentine. Cet objectif général se divisait en trois objectifs spécifiques :

1. Dresser un constat de la présence et du rayonnement des poètes québécois sur la scène littéraire hispano-américaine en établissant une bibliographie commentée des traductions publiées sous forme de recueils et d'anthologies;

2. Rendre ces résultats accessibles par la création et la mise en ligne d'une base de données pouvant servir à l'élaboration de projets de recherche et de traduction;

3. Cerner l'image de la culture et de la langue québécoises que véhiculent les traductions en étudiant les contextes de production, de diffusion et de réception, ainsi que le contenu d'anthologies représentatives des tendances observées.

Le présent article se concentrera sur le deuxième objectif, qui visait la création d'une base de données. En 2013, nous avons mis en ligne un moteur de recherche accessible gratuitement

\footnotetext{
2 «Il n’y a toujours pas de marché au Mexique pour la littérature canadienne : on ne l’a pas encore assez publicisée ». (Nous traduisons).
} 
qui répertorie de façon à la fois juste et exhaustive les traductions espagnoles de poésie québécoise de langue française parues en Amérique latine de 1961 à 201133. En 2017, Laurent Aussant, candidat à la maîtrise en études langagières de l'Université du Québec en Outaouais (UQO), a procédé à une mise à jour exhaustive des données pour inclure les traductions parues de 2010 à 20154. Les deux bases de données du site Web TEPOQAL ont été conçues au moyen de Tiki Wiki, un logiciel libre de gestion de contenu et de travail collaboratif. L'une contient une bibliographie exhaustive des anthologies collectives et recueils individuels traduits; l'autre, un répertoire des poèmes inclus dans les anthologies. Ces bases constituent de précieuses sources d'information pour traducteurs, traductologues et comparatistes et contribuent à la diffusion internationale de la poésie québécoise.

En 2014, Lane-Mercier déplore l'absence d'un «répertoire complet et à jour des œuvres littéraires canadiennes traduites dans les deux langues officielles » (2014b, p. 518). Au moment où nous avons créé ces bases de données, on était donc loin d'avoir accès à un répertoire qui recenserait les traductions d'œuvres canadiennes en langues étrangères. Il n'existait non plus aucun moteur de recherche accessible en ligne gratuitement qui répertoriait avec exactitude l'entièreté des traductions espagnoles de la poésie québécoise de langue française parues en Amérique latine ${ }^{5}$. Pour trouver les traductions, on devait passer au peigne fin beaucoup de bases de données et catalogues de bibliothèques, les pages Web de nombreuses maisons d'édition, voire les sites de librairies virtuelles. Or, comme nous le verrons plus tard, ce n'est pas parce qu'une référence apparaît dans un catalogue qu'elle est nécessairement fiable. Par ailleurs, le fait de trouver une référence ne signifie pas que l’on

\footnotetext{
${ }^{3}$ On peut consulter le moteur de recherche à l'adresse suivante $:$ http://linguistech.ca/tepoqal.

${ }^{4}$ Nous le remercions d'ailleurs pour son apport à la révision du présent article.

${ }^{5}$ Les professeurs Luise von Flotow, Marc Charron et Hugh Hazelton ont dirigé, entre-temps, un projet de recherche ayant mené à la création d'une base de données répertoriant des œuvres littéraires canadiennes (tous genres confondus), traduites en Amérique Latine : http://artsites.uottawa.ca/canada-latin-america/fr/research-project/. Cela dit, la base de données ne semble plus accessible sur le Web à l'heure actuelle.
} 
trouve le livre lui-même, et il faut parfois l'avoir entre les mains pour confirmer les informations trouvées sur le Web. Notons qu'entre 2010 et 2013, où nous avons effectué les recherches bibliographiques ayant mené à la création des bases de données TEPOQAL, Gillian Lane-Mercier n'avait pas encore partagé les fruits de ses investigations bibliographiques sur la littérature anglo-québécoise en traduction française, qui ont posé le même genre de défis que ceux auxquels nous avons été confrontés (2014a et 2014b). Chose certaine, nous constaterons ci-dessous à quel point Lane-Mercier a raison de remarquer que « la non-fiabilité des banques de données à des fins de recherche n'est pas propre au champ canadien » (2014b, p. 519).

Nos recherches documentaires menant à la création d'une base de données s'inscrivent dans un courant qui prend de l'ampleur au Canada en traductologie, notamment depuis les recherches effectuées à l'Université de Sherbrooke dans le but de mettre en ligne une bibliographie exhaustive des écrits traductologiques au Canada (Grant et Mezei, 2007), laquelle n'est malheureusement plus accessible. Lane-Mercier insiste d'ailleurs toujours sur la nécessité de créer des bases de données bibliographiques à la fois ciblées et exhaustives, dans la veine de la Bibliographie que Philip Stratford avait fait paraître en 1972, qui

a eu un effet d'autant plus retentissant sur les recherches traductologiques alors en voie d'émergence au Canada anglais et au Québec qu'elle a rendu possibles des analyses quantitatives et qualitatives jusqu'alors inexistantes, faute d'accès autre que partiel à des données ainsi réunies (2014a, p. 534).

Non seulement nous sommes d'accord avec Lane-Mercier, mais nous croyons en outre essentiel de ne pas s'arrêter à la présentation des critères ayant mené à la constitution de telles bibliographies, mais d'inclure aussi, comme Grant et Mezei l'ont fait, une discussion liée à la création d'un outil électronique permettant à d'autres chercheurs d'accéder facilement et gratuitement aux données répertoriées. 
Après avoir expliqué les critères d'inclusion des poètes et des publications dans notre corpus, nous présenterons la genèse du premier des deux outils, intitulé TEPOQAL I, un acronyme pour: Traductions Espagnoles de la POésie Québécoise en Amérique Latine. Nous traiterons des défis que nous avons relevés lors de la collecte de données et de la conception de la base de données et nous les comparerons à ceux rencontrés par les créateurs de la Bibliographie d'études comparées des littératures canadienne, québécoise et étrangèré, pionniers en diffusion électronique de contenu bibliographique (Grant et Mezei, 2007). Notre expérience révèlera, d'une part, que les informations bibliographiques accessibles sur le Web sont à la fois surabondantes et insuffisantes et, d'autre part, qu'il est aujourd'hui po ssible pour des chercheurs formés en lettres et sciences humaines n'ayant pas de connaissances poussées en informatique de construire eux-mêmes leur propre outil de diffusion.

\section{Critères d'inclusion de poètes et de titres}

Selon Poupaud, Pym et Torrres Simón, les recherches bibliographiques sont régies par trois principes de base : 1) l'ubiquité des traductions; 2) les filtres des concepteurs des répertoires existants; 3) les filtres des chercheurs qui utilisent ces répertoires (p. 265-269). Ainsi, le fait qu'il y ait des traductions partout rend impossible une réelle exhaustivité de leur compilation : il faut donc procéder à des choix. L'accessibilité à l'information est déterminée, dans un premier temps, par les outils que les chercheurs ont à leur disposition, influencés par les filtres (ou critères d'inclusion) de leurs concepteurs, qui peuvent être objectifs ou non. À cet égard, Sapiro souligne que « la question n'est [donc] pas seulement "comment utiliser ces données?" mais “qui produit quoi et pour qui?” "(p.46). Dans un deuxième temps, les

\footnotetext{
6 L'URL était autrefois http://compcanlit.usherbrooke.ca/index.html, mais le site n'est aujourd'hui
} malheureusement plus accessible. 
chercheurs ont eux aussi leurs propres «filtres », qui peuvent ou non coïncider avec ceux des concepteurs des outils existants. Ultimement, Poupaud, Pym et Torres Simón conseillent aux chercheurs d'opter pour des filtres restreints et « pragmatiques » (p. 269), ce que nous avons fait en nous limitant à la poésie québécoise d'expression française traduite en espagnol, en Amérique latine, et publiée sous forme de livres (recueils et anthologies). Comme l'explique Lane-Mercier (2014b, p. 523), «les compilations de données empiriques ne sont jamais neutres », ce dont nous étions d'ailleurs conscients au moment d'entamer nos recherches. Depuis la «préhistoire » du projet en 2006, les critères d'inclusion des poètes et des titres dans la bibliographie ont été recadrés à trois reprises. En 2006, nous avions recensé 11 anthologies collectives de poésie traduites en espagnol, parues en Espagne ou en Amérique latine entre 1966 et 2006 ainsi que 52 recueils individuels traduits, parus entre 1980 et 2005 (Stratford, 2008). Ces recherches préliminaires avaient démontré que c'est surtout en Amérique latine que paraissent les traductions espagnoles de poésie québécoise et presque exclusivement au Mexique. En fait, la proportion des versions espagnoles parues dans la péninsule ibérique est infime. Peut-être en raison des relations politiques entre le Québec et la Catalogne (Córdoba Serrano, 2007, p. 777), la majorité des traductions publiées en Espagne ne sont pas vers l'espagnol (le castillan), mais vers le catalan.

Des recherches plus poussées ont révélé que la majorité des traductions de poésie québécoises en espagnol avaient été produites au Mexique à partir de l'an 2000, souvent en coédition entre les Écrits des Forges au Québec et Mantis Editores, et que la majorité était parue en lien avec la FIL de 2003, où le Québec était l'invité d'honneur (Stratford, 2013a et 2013b). En outre, les échanges éditoriaux semblaient le fruit de contacts personnels initiés dans les années 1980 par des poètes québécois et mexicains (Stratford, 2013b). Pour sa part, l'étude des anthologies avait permis de constater, entre autres, l'omniprésence de la notion de 
nationalisme québécois et de l'affirmation d'une «latinité » commune entre le Québec et le Mexique (Stratford, 2013c et 2014). L'Argentine, loin derrière le Mexique, se démarquait comme deuxième pays de publication, mais de façon mineure. Ainsi, pour la poursuite des recherches, nous avons choisi de concentrer nos efforts sur les publications en sol latinoaméricain, en particulier au Mexique, pays hispanophone d'Amérique du Nord où est publiée la majorité des versions, et en Argentine, principal pôle de diffusion en Amérique du Sud.

Le choix de nous pencher sur la littérature québécoise spécifiquement, plutôt que canadienne, ou même franco-canadienne, est lié au fait que le Québec s'est doté assez tôt d'une «politique culturelle précisant la volonté du gouvernement d'affirmer l'identité culturelle québécoise par rapport aux autres cultures nationales et de soutenir les entreprises, les organismes et les artistes qui excellent dans leur domaine et veulent s'inscrire dans les grands courants internationaux 7 », laquelle a encouragé la traduction d'œuvres québécoises. La poésie québécoise traduite en Amérique latine, en particulier sous la forme d'anthologies, semble avoir servi de vecteur pour diffuser une idéologie séparatiste (Stratford, 2013c et 2014). En 2006, nous avions retenu sous l’appellation «poètes québécois francophones » les Québécois d'origine ou d'adoption identifiés à ce genre sur les sites de l’Union des écrivaines et des écrivains québécois (UNEQ) et de l'Infocentre littéraire des écrivains québécois (ÎLE). Nous avions aussi considéré les noms répertoriés dans les principaux ouvrages traitant de poésie québécoise, signés François Dumont (1999), Laurent Mailhot (1997) et Pierre Nepveu (1990), ainsi que François Ouellet et Hans-Jürgen Greif (2004). Il s'agissait à l'époque d'un bon point de départ, mais à l'heure d'approfondir les recherches en 2010, nous ressentions le besoin d'élargir les critères d'inclusion, tout en les systématisant. Pour ce faire, nous avons

\footnotetext{
${ }^{7}$ Voir Ministère des affaires culturelles - Direction des communications (1992), La politique cultur elle du québec : notre culture, notre avenir, Québec, Gouvernement du Québec, p.7, https://www.mcc.gouv.qc.ca/fileadmin/documents/publications/politiqueculturelle1992_complet_ROC.pdf.
} 
emprunté une méthodologie semblable à celle adoptée par Maria Sierra Córdoba Serrano sur les traductions d'œuvres de fiction québécoises en Espagne. Dans le cadre de ses recherches, Córdoba Serrano disait avoir :

inclus non seulement les auteurs canadiens d'origine québécoise [...], mais aussi les auteurs d'origine non québécoise, pourvu que ces auteurs aient pris position dans le champ littéraire québécois, en publiant des articles dans des revues littéraires ou dans des journaux québécois, en assistant à des colloques littéraires au Québec, en étant membre de l'Union des écrivaines et des écrivains québécois, etc. (p. 765).

Le concept de «prise de position dans le champ littéraire québécois » a été particulièrement utile pour traiter les cas d'exception. Ont ainsi été exclus de l'étude les poètes francophones qui publient au Québec, mais qui ne font pas partie de l'UNEQ ou qui ne sont pas répertoriés sur le site de l'ÎLE et qui ne se présentent pas comme des poètes québécois. Michel A. Thérien, par exemple, n’a pas été retenu. Certes, il a publié un recueil aux Écrits des Forges, mais la maison d'édition le présente comme un "Franco-ontarien [sic] de naissance ${ }^{8}$ », et il faisait partie en 2016 des poètes franco-ontariens primés par la bibliothèque publique de Toronto ${ }^{9}$. Il n'est pas non plus membre de l'UNEQ ni répertorié sur le site de l'ÎLE. Par contraste, Patrice Desbiens, né à Timmins en Ontario, habite Montréal depuis des années. Il est répertorié comme auteur québécois sur le site de l'ÎLE, où l'on dit qu'il est membre de l'UNEQ. Il déclare même en entrevue avec Le Devoir en 2015 : «Il y a du monde qui pense encore que je vis ce qui est dans Sudbury. Ce n'est plus mon monde, ça. [...] J'étais là dans le temps, faque j'écrivais ce que je voyais. Maintenant, je suis ici, faque j'écris ce que je vois ici. » (Tardif, 2015, en ligne) On peut donc conclure que tout en étant toujours un «poète franco-ontarien», il «a pris position dans le champ littéraire québécois ». C’est le cas également des écrivains migrants

\footnotetext{
8 Voir Écrits des Forges, Description de l'œuvre Cuerpo Salvage/Corps sauvage de Michel A. Thérien, http://www.ecritsdesforges.com/oeuvre.php?id=891.

9 Voir Toronto Public Library (2016), "Les auteurs franco-ontariens à l'honneur: Michel A. Thérien », http://torontopubliclibrary.typepad.com/en francais/2016/08/les-auteurs-franco-ontariens-\%C3\%A0-lhonneurmichel-a-.html.
} 
comme Anthony Phelps, qui est lui aussi répertorié sur le site de l'ÎLE et a publié l'essentiel de son œuvre au Québec.

Dans l'immédiat, la recherche n'inclut pas les auteurs bilingues originaires ou résidants du Québec qui écrivent leur poésie ou s'autotraduisent aussi bien en français qu'en espagnol : leur œuvre est fort intéressante, mais difficile à traiter dans le cadre des présentes recherches, car il est ardu, dans de tels cas, de départager clairement les langues source et cible. À cet égard, le cas de Françoise Roy, poète et traductrice née au Québec qui vit au Mexique depuis 1992, est particulièrement digne de mention. Roy écrit, traduit et s'autotraduit en français comme en espagnol. Elle est active sur la scène littéraire québécoise comme mexicaine : entre autres, elle a remporté en 2007 le Prix National de Poésie Alonso Vidal au Mexique et, au Québec, elle a été finaliste en 2009 aux Prix littéraires de RadioCanada dans la catégorie poésie. En fait, son cas est si exceptionnel qu'il mériterait, à lui seul, une étude à part entière. En effet, même après avoir communiqué avec elle, nous ne sommes toujours pas en mesure de savoir avec certitude lesquels de ses recueils ont d'abord été rédigés en français puis traduits en espagnol. Pour l'instant, nous n'avons pu intégrer qu'un de ses recueils à notre corpus, Le voile premier/El velo uno (Roy, 2003), car une note des Écrits des Forges précise qu'elle « en a elle-même fait la traduction en espagnol 10 ».

Bien que nous n'excluions pas la possibilité d'élargir les paramètres de l'étude pour ajouter au corpus les poètes québécois anglophones et allophones, bilingues ou multilingues, en traduction ou en autotraduction espagnole, nous nous penchons exclusivement, pour l'instant, sur les traductions du français (langue de départ) en espagnol (langue d'arrivée) de poésie québécoise publiées ou distribuées en Amérique latine de 1980 à 2011. Le critère de lieu de distribution nous a permis d'inclure les ouvrages parus au Québec ou aux États-Unis

\footnotetext{
${ }^{10}$ http://www.ecritsd esforges.com/oeuvre.php?id=751.
} 
qui étaient destinés de façon explicite à un public latino-américain. C'est le cas, par exemple, de l'Antología del Noroît (Imbert, 1998) et des plaquettes de Pen Press (Audet, 2010 et Dorion, 2003), éditées respectivement à Montréal et à New York pour être distribuées principalement en Argentine.

Comme la liste de poètes à considérer, les formats de publication à inclure ont fait l'objet d'un recadrage en 2010. Quatre ans plus tôt, les premières recherches visaient à ratisser autant de traductions espagnoles que possible publiées sur support papier, en Amérique latine comme en Espagne, sous forme de recueils, d'anthologies et de sélections anthologiques parues dans des revues littéraires. L'idée maîtresse était de brosser un panorama des traductions existantes. Or, ces recherches ont révélé qu'il était très difficile de trouver les sélections traduites parues dans des revues: il est ardu d'obtenir une liste complète et fiable de toutes les revues latino-américaines susceptibles d'avoir publié des traductions espagnoles de poésie québécoise et, même lorsque l'on possède une telle liste, il n'est souvent pas possible de consulter virtuellement le contenu des revues. Puisque la base de données projetée se voulait à la fois fiable et exhaustive, il a été décidé de privilégier les publications sous forme de livres : les recueils individuels et les anthologies collectives.

\section{La collecte de données : le piège de la toile}

Comme l'explique Lane-Mercier, un répertoire bibliographique «complet» se doit d'être « exhaustif», «systématique» et «sans erreur» (2014b, p. 518-519). Ces trois critères justifient, d'une part, la sélection d'un corpus restreint (Lane-Mercier 2014b, p. 527) et la consultation de plusieurs sources croisées (Sapiro, 2008, p. 56-57). Dans le cas de la poésie, qui implique souvent de petites maisons d'édition et des tirages limités, qui plus est de la culture québécoise, qui se situe en périphérie du polysystème canadien, un outil comme 
l'Index Translationum de l'UNESCO n'aurait été d'aucune utilité. En effet, expliquent Poupaud, Pym et Torres Simón, l'Index ne convient pas à l'étude de livres à faible diffusion ni à des corpus spécifiques (p. 264), sans compter qu'il ne permet pas d'effectuer une recherche par genre. Le point de départ de la recherche a donc été la liste de poètes québécois déjà traduits vers l'espagnol en Amérique latine que nous avons élaborée en 2006, puis mise à jour en 2007, ainsi qu'une liste de poètes qui, à notre connaissance, ne l'avaient pas encore été. Chaque nom a fait l'objet d'une recherche exhaustive sur Internet au moyen de trois principaux outils: WorldCat 11 , un métacatalogue réunissant le contenu de milliers de bibliothèques publiques et universitaires de partout dans le monde; L'infocentre littéraire des écrivains québécois (ÎLE) ${ }^{12}$, un site Web contenant des renseignements biobibliographiques sur quelque 1000 écrivains québécois; et Google (recherche avancée13), un des plus populaires moteurs de recherche sur Internet. Nous avons aussi consulté les catalogues Amicus de Bibliothèque et Archives Canada14 et Iris de Bibliothèque et Archives nationales du Québec ${ }^{15}$ ainsi que les sites Web des maisons d'édition québécoises et latino-américaines le plus souvent impliquées dans des projets de traduction espagnole de poésie québécoise ${ }^{16}$. Nous évoquerons ici les avantages de ces différents outils, mais aussi les problèmes rencontrés qui, nous semble-t-il, sont propres à l'ère numérique et au contexte de mondialisation ${ }^{17}$ actuel.

\footnotetext{
11 WorldCat, http://www.worldcat.org/.

12 L'infocentre littéraire des écrivains québécois, http://www.litterature.org/.

${ }^{13}$ Google (recherche avan cée), http://www.google.ca/advanced search.

14 Bibliothèque et Archives Canada, Amicus, catalogue national canadien, http://amicus.collectionscanada.gc.ca/aaweb/aaloginf.htm.

15 Bibliothèque et Archives nationales du Québec, catalogue Iris, http://iris.banq.qc.ca/iris.aspx.

${ }^{16}$ Entre autres, mentionnons Les Écrits des Forges (http://www.ecritsdesforges.com/) et les Éditions du Noroît (http://www.lenoroit.com/) du côté québécois, et, du côté mexicain, Aldus (http://alduseditorial.tumblr.com/), Literalia Editores (http://literaliaeditores.info/), Mantis Editores (http://mantiseditores.com/), Plan C Editores (http://planceditores.com/) et les presses de l'Université nationale autonome du Mexique (UNAM) (http://www.libros.unam.mx/). Toutefois, la plupart de ces sites ne semblent plus actifs.

17 Nous utilisons le terme "mondialisation » au sens où l'entend Sapiro, soit «circulation mondiale des biens culturels » (p.8).
} 


\section{Les recherches sur WorldCat}

WorldCat est une base de données regroupant plus de 10000 catalogues de bibliothèques d'ici et d'ailleurs. Elle nous a donné accès à des bibliothèques dont nous n'aurions pu connaître l'existence autrement, y compris en Amérique latine, ce qui représentait un grand avantage dans le cadre de notre recherche. Plusieurs fonctions de WorldCat nous ont été très utiles. Par exemple, la base de données repère la bibliothèque la plus proche disposant de la publication désirée, selon l'emplacement de l'utilisateur. Dans chaque fiche bibliographique figurent aussi les liens vers les catalogues d'origine.

Dans WorldCat, nous avons fait une recherche par nom d'auteur pour chaque poète de la liste de départ, en restreignant les résultats à ceux dont la langue était l'espagnol puis à ceux dont la langue était non déterminée. Ainsi, nous avons pu constater que plusieurs publications unilingues espagnoles ou bilingues (français-espagnol) étaient rangées à la fois dans la catégorie « espagnol » et dans « langue non déterminée ». Il nous arrivait donc de nous retrouver avec deux fiches pour un seul livre. De plus, les liens vers les catalogues d'origine étaient parfois invalides ou ne menaient pas à l’ouvrage désiré. Par exemple, il existe une fiche pour la traduction espagnole du recueil Grand hôtel des étrangers de Claude Beausoleil (2003). Or, le lien de Bibliothèque et Archives Canada mène vers la fiche du recueil original et non vers celle de la version espagnole, qui se trouve pourtant dans le catalogue.

\section{Les recherches sur l'îLE}

Après ces premières recherches dans WorldCat, nous avons consulté le site de l'ÎLE. Dans la page d'accueil, on précise que le site recense les publications d'auteurs québécois « dans toutes les langues et dans tous les pays », et qu'un partenariat avec Bibliothèque et Archives nationales du Québec «garantit l'exactitude des informations publiées». Toutefois, il est 
précisé que "seuls les livres à auteur unique sont recensés», ce qui exclut les ouvrages collectifs comme les anthologies. De plus, il appert que ce sont les auteurs eux-mêmes qui fournissent les renseignements au sujet de leurs œuvres traduites. Le site est donc apparu comme une source potentielle sinon d'anthologies traduites du moins de recueils individuels traduits.

Néanmoins, peut-être parce que les références bibliographiques des traductions sont fournies "grâce à la collaboration des auteurs », certaines sont incomplètes. Notons aussi qu'elles sont rarement présentées en ordre chronologique. Il arrive qu'un ou plusieurs titres traduits manquent à l'appel, comme Les pyramides du cœur de Bernard Pozier (2009). Enfin, il n'est pas rare que ce soit le titre français plutôt que l'espagnol qui identifie la référence, comme pour le recueil Vertige de l'avant-scène de Brossard (2000), si bien qu'il faut lire la description entière pour savoir qu'il s'agit en fait d'un recueil traduit. Cela dit, l'ÎLE nous a permis de trouver quelques perles, comme la version espagnole de Songe que je bouge de Gilles Cyr, signée Benjamín Valdivia (2001). En fait, la quantité d'informations au sujet des versions espagnoles sur le site de l'ÎLE semble dépendre de la connaissance que le poète a de l'existence des versions (et peut-être de sa maîtrise de la langue d'arrivée) et de son désir de contribuer à leur diffusion.

\section{Les recherches sur Google}

Même si l'information trouvée dans WorldCat et l'ÎLE était parfois incomplète, ces sites ont quand même constitué des sources utiles pour l'objet de notre étude : très conviviaux, ils présentent l'information de manière organisée; le tri et l'évaluation des données se sont donc faits rapidement et facilement, ce qui n'a pas été le cas pour l'information trouvée sur la grande toile. Au moyen de Google, nous avons fait une recherche avancée par nom d'auteur, 
en restreignant la langue des résultats à l'espagnol. Nous nous sommes rapidement rendu compte qu'il fallait écarter une grande quantité de pages Web non pertinentes ou non fiables en raison de plusieurs facteurs, comme le fait qu'un même nom peut référer à diverses personnes. Par exemple, André Roy est un poète québécois qui partage son nom avec un joueur de hockey de la Ligue nationale. Ainsi, les résultats comptaient plus de 50 pages, dont seulement une minorité concernait l'auteur.

En outre, l'engouement pour les médias sociaux et la prolifération de pages personnelles font augmenter le nombre de renseignements à traiter. En effet, nous avons souvent trouvé des traductions de poèmes québécois publiées par des internautes sans mention du nom du traducteur; c'est le cas de textes d'André Roy ${ }^{18}$ et d'Émile Nelligan (Sabido Sánchez, 2012). Enfin, des renseignements bibliographiques publiés sur des pages personnelles nous ont mis sur de fausses pistes. Par exemple, nous avons cru à tort que le recueil Orchidée nègre d'Anthony Phelps avait été traduit. En effet, un internaute a écrit que ce livre, publié par la maison d'édition Plural, était le seul de l'auteur qui avait été traduit vers l’espagnol dans son intégralité19. Or, nos recherches ont révélé que Plural est en fait une revue mexicaine. Nous avons consulté la publication à la bibliothèque de l'Université d'Ottawa pour nous rendre compte qu'un seul poème traduit de Phelps y avait été publié.

Un autre phénomène lié à l'avènement des nouvelles technologies nous a paru problématique : en faisant une recherche sur Jean-Éric Riopel, nous avons trouvé un article espagnol de l'Encyclopedia Encydia ${ }^{20}$ portant sur le prix Émile-Nelligan ${ }^{21}$. Selon cette page, Jean-Éric Riopel aurait été lauréat du prix pour un recueil intitulé Mariposas rebeldes. Nous connaissions l'existence d'une version espagnole du recueil Papillons réfractaires sous le titre

\footnotetext{
$18 \mathrm{http}$ //holamellamokarla.tumblr.com/post/11157273809/circosdeintimidad-andre-roy. La page n'existe plus aujourd'hui.

${ }^{19} \mathrm{http}: / /$ www.elhablador.com/blog/2010/01/17/laurel-machete-3/. La page n'existe plus aujourd'hui.

${ }^{20} \mathrm{http}: / /$ es.encydia.com/index. La page n'existe plus aujourd'hui.

${ }^{21} \mathrm{http}$ ://es.encydia.com/fr/Precio \%C3\%89mile-Nelligan. La page n'existe plus aujourd'hui.
} 
Mariposas refractadas (Riopel, 2004), mais avons pensé qu'il y en avait peut-être une deuxième. Nous avons cependant remarqué bon nombre d'anomalies d'ordre linguistique sur la page : par exemple, le nom de la maison d'édition Écrits des Forges avait été traduit par Escritos de las forjas et le nom de la maison d'édition Les Intouchables avait été traduit par Los Intouchables. De plus, la structure de l'article de l'Encyclopedia Encydia correspondait en tous points à celle de l'article français de Wikipédia, et une note de bas de page explique dans un amalgame multilingue incompréhensible: «This Página is based in año artículo from Wikipedia [sic] ». Nous avons émis l'hypothèse que la page d'Encydia était probablement une traduction automatique de celle-ci. Nous avons trouvé une note au début de quelques pages d'Encydia qui confirme que l'encyclopédie contient effectivement des pages traduites de manière automatique : «El artículo necesita algunas mejoras de traducción. Puede ser una traducción automática o contener fragmentos sin traducir 22 ». De plus, dans la page du site pearltrees.com ${ }^{23}$ illustrant le réseau des encyclopédies Web, Encydia apparaît comme une branche de Wikipédia, au même titre que Portail et Visual Dictionary. Il reste cependant que le Web est, curieusement, avare de renseignements au sujet d'Encydia: on n'y trouve aucune information sur les concepteurs de l'encyclopédie, les sources des articles ni sur le type de logiciel de traduction automatique employé.

Pour l'instant, la possible traduction automatique de contenu de pages Web ne pose pas de graves problèmes au traductologue, puisque la qualité linguistique douteuse des informations bibliographiques incite à douter de leur fiabilité. Les chercheurs peuvent donc facilement arriver à faire la distinction entre la référence bibliographique d'un recueil traduit

\footnotetext{
${ }^{22}$ La traduction de cet article nécessite une révision. Il peut être le fruit d'une traduction automatique ou contenir des fragments encore non traduits. (Nous traduisons). La mention se trouvait notamment sur les pages http://es.encydia.com/ca/Cristianism o; http://es.encydia.com/ca/Hamburguesa et http://es.encydia.com/ca/Alquimia, qui n'existent plus aujourd'hui.

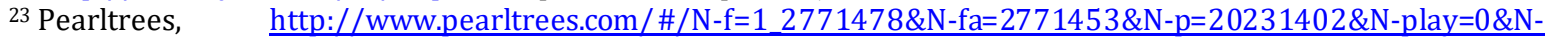
$\underline{\mathrm{s}=1 \_2771478 \& \mathrm{~N}-\mathrm{u}=1 \_278063}$.
} 
et la traduction automatique de la référence du recueil original. Cependant, avec les progrès constants dans le domaine des technologies langagières, il se pourrait qu'à moyen ou à long terme, la qualité de la traduction automatique des références s'améliore au point de brouiller les pistes, ce qui pourrait faire croire qu'un recueil a été traduit, alors que ce n'est pas le cas.

\section{Les autres pistes de recherche}

Au cours de nos recherches, nous avons aussi consulté les pages Web de maisons d'édition québécoises et mexicaines, qui contenaient parfois un catalogue de leurs publications. Ces derniers auraient pu constituer des sources au moins aussi fiables que les catalogues de bibliothèques. De manière surprenante, toutefois, certains de ces catalogues ont plutôt compliqué notre tâche. Par exemple, celui du site des Éditions des Forges, maison d'édition ayant publié une majorité des publications faisant partie de la base de données TEPOQAL, contient des informations qui contredisent parfois celles retrouvées dans les livres euxmêmes. Par exemple, le recueil Poésie Mexique-Québec Tome I a été publié en 2008 (Beausoleil), mais figure dans le catalogue de 2010 sur le site des Écrits des Forges. De plus, lorsqu'il nous était impossible de consulter un livre, nous pensions facilement trouver la ville et le pays d'édition sur les sites d'éditeurs mexicains. Or, à quelques reprises, seule une adresse courriel était donnée, comme dans le cas des Ediciones Sin Nombre, si bien que nous avons dû chercher ces renseignements ailleurs. Confrontés dans certains cas à des renseignements bibliographiques lacunaires ou contradictoires, nous avons établi un critère : les renseignements bibliographiques devraient être appuyés par non pas une, mais deux sources que nous jugions fiables (par exemple les catalogues de bibliothèques et les bibliographies). 
En dernière instance, malgré toute l'information que nous pouvions trouver en ligne, que ce soit sur les bases de données et les catalogues en ligne ou sur Google, le recours à Internet ne semblait pas suffisant pour parachever de façon adéquate la mise à jour de notre bibliographie. Dans les cas où nous n'arrivions pas à valider les informations au moyen de deux sources fiables, nou s avons eu recours à d'autres modes de recherche : 1) la consultation directe de la publication lorsque c'était possible grâce au prêt entre bibliothèques ou à un achat en ligne, et 2) la communication avec les auteurs, les traducteurs ou les maisons d'édition. Par exemple, c'est grâce au prêt entre bibliothèques que nous avons pu avoir accès à l'anthologie El teatro de las emociones, livre rare réunissant des poèmes et des œuvres d'art visuel (Méndez, 2004). La plaquette de 23 pages est parue à Cuba en 2004 et n’a été tirée qu’à 20 exemplaires. La notice de Bibliothèque et Archives Canada (reproduite sur WorldCat) laissait entendre que le livre était une publication bilingue: «texte en espagnol et en français». Lorsque nous avons pu consulter le livre, nous avons constaté qu'il est effectivement dans les deux langues, mais que les textes sélectionnés n'apparaissent qu'en langue originale: soit en espagnol (pour les auteurs cubains), soit en français (pour les auteurs québécois). L'anthologie ne contient donc aucun texte traduit, et elle a dû être retirée du corpus.

Évoquons aussi le cas d'une publication de France Théoret qui nous a semblé intrigant. Nous avons trouvé deux références à une version espagnole de Bloody Mary (1981), l'une dans une bibliographie publiée par Hélène Girard (1988) dans Voix et Images, l'autre dans la Bibliographie d'ouvrages en études québécoises en langue espagnole ${ }^{24}$, mise en ligne par l'Association internationale des études québécoises, qui a toutefois été retirée lors de la récente refonte du site. Ces références laissaient entendre que des extraits du recueil avaient

\footnotetext{
${ }^{24} \underline{\mathrm{http}}: / /$ www.aieq.qc.ca/publications/bibliographie.espagnol.2006.pdf. La page n'existe plus aujourd'hui.
} 
été traduits en espagnol dans un livre intitulé La bolsa y la vida ediciones. Pourtant, nos recherches indiquaient que ce « titre » correspondait en fait au nom d'une maison d'édition et non au titre d'un ouvrage. Nous avons essayé en vain de communiquer par courriel avec les fondateurs des éditions La bolsa y la vida, puis avons communiqué avec France Théoret. Celleci a eu l'amabilité de nous envoyer une copie de la page contenant la traduction, qui aurait été publiée en 1981. Après réception du document, il était clair que celui-ci n'entrait pas dans notre corpus, puisqu'il ne s'agit pas d'un livre, mais de quelques extraits traduits, parus sur un genre de lithographie artistique. Cela dit, même avec cette «affiche » en mains, des doutes persistent encore sur la nature exacte de la publication : s'agit-il d'un extrait de revue, d'un extrait d'anthologie ou même d'une traduction intégrée à un genre de livre-objet? Selon une définition de Claudette Hould, le livre-objet est une œuvre dont la vocation est principalement plastique (Hould citée par Bernier, 1986, p. 528). L'hypothèse du livre-objet est plausible, car quelques titres que nous pensions originellement être des recueils de poèmes en espagnol font en fait partie d'œuvres d'art visuel et ne correspondent donc pas aux paramètres de notre corpus. Par exemple, les versions espagnoles de poèmes de Pierre Morency et de Claude Beau soleil ont été intégrées dans des œuvres de l'artiste visuel René Derouin ${ }^{25}$.

Au cours de l'hiver 2017, nous avons également découvert un article de Françoise Roy paru le 13 janvier 2016 dans Blanco Móvil, où la traductrice dit avoir produit une version française du recueil Ne calme pas les dragons de Jean-Marc Desgent pour publication en coédition québécoise et mexicaine. Or, nous n’avons trouvé aucune autre trace de ce recueil : aucune référence, ni année de publication, ni ISBN. La page Facebook des Éditions de la Grenouillère le mentionne, mais leur lien renvoie à cette même page de Blanco Móvil. Nous avons donc contacté les Éditions de la Grenouillère, qui nous ont répondu qu'ils n'avaient

\footnotetext{
${ }^{25}$ Voir WorldCat, http://www.worldcat.org/oclc/49123830.
} 
pour leur part que la version française du recueil. Ils nous ont recommandé de contacter l'éditeur mexicain, Literalia editores, lequel ne nous a jamais répondu. Nous en avons conclu que ce projet de traduction ne s'est jamais matérialisé.

D’après nous, le genre de recherche universitaire que nous avons effectuée sur le Web illustre très bien l'effet pervers de l'explosion de l'information mise à la disposition des internautes : la quantité des renseignements est certes impressionnante; le travail de tri et d'évaluation de la pertinence, de la fiabilité et de l'exactitude de l'information est, par contre, tout aussi colossal. En cette ère de surabondance de renseignements en tout genre publiés à diverses fins sur Internet, TEPOQAL est une base de données qui simplifie le travail des chercheurs : les fiches bibliographiques contiennent les renseignements les plus complets et les plus fiables possible, préalablement triés dans l'optique de la recherche scientifique.

\section{La diffusion des données : genèse de TEPOQAL}

Le principal objectif de cette dernière étape de notre projet de recherche consistait à créer une base de données bibliographiques en ligne exhaustive, fiable et librement accessible afin de permettre aux chercheurs, professeurs, traducteurs, écrivains, étudiants et au grand public de consulter l'information gratuitement. Même si nous disposions à l'époque d'une bibliographie statique de 100 titres $^{26}$, le passage des titres d'un fichier de traitement de texte à une base de données n'était pas chose facile. Pour une équipe de recherche en lettres, sans formation ni connaissances approfondies en informatique, il s'agissait d'un défi de taille que d'avoir à adapter un outil informatique pour répondre à des besoins bien définis, sans pour autant connaître cet outil.

${ }^{26}$ En date du 20 mars 2018, TEPOQAL I compte 119 titres. 
Le site Web www.compcanlit.ca (CCL) de la Bibliographie d'études comparées des littératures canadienne, québécoise et étrangères, qui n'existe plus aujourd'hui, a été notre première référence. John Taylor-Johnston expliquait en 2004 que la tentative de mise en ligne initiale de la bibliographie CCL en 1995 n'avait pas abouti parce que « les chercheurs n'étaient pas prêts à composer avec la technologie requise », mais que le recours en 2002 à un « modèle de recherche à code source libre (Open Source Initiative) » avait pu remédier au problème (p. 177). Convaincus par ce modèle et dans la même logique de libre diffusion de l'information et du libre accès, nous savions que TEPOQAL devait être conçue à l'aide d'un logiciel ouvert. Dix ans après la création de CCL, la technologie ne s'est pas transformée de manière radicale, mais le cadre théorique a changé significativement, car la mondialisation a amené de nouveaux modes de diffusion de l'information. Nous présenterons ici les étapes de la création de notre base de données bibliographiques en ligne, depuis ses débuts jusqu'à son aboutissement.

\section{La solution : Tiki Wiki}

Pour notre équipe inexpérimentée dans les domaines des technologies de l'information et de la programmation, il n’a pas été facile de distinguer entre besoins, possibilités et désirs lors de la création de la base de données en ligne. Nous avions du mal à réfléchir en termes binaires et à prendre des décisions adéquates. Nous avons donc demandé conseil au Centre de recherche en technologies langagières (CRTL), alors logé à même les locaux de l'UQO27, qui avait une expertise dans l'utilisation de la technologie dans le domaine des langues et de la traduction et menait déjà des projets interdisciplinaires multilingues. À notre grande surprise, le CRTL nous a suggéré de concevoir nous-mêmes la base de données et nous a offert de l'héberger sur

\footnotetext{
${ }^{27}$ Le CRTL est depuis devenu le Cilex : $\underline{\text { https://cilex.ca/. }}$
} 
Linguistech ${ }^{28}$, « un site Web de référence pour les langagiers, conçu dans l'esprit du “tout sous une même adresse" et dans une visée de collaboration professionnelle et pédagogique » (Rivet, n. d., p. 7). Avec l'aide et la patience de l'équipe du CRTL, nous avons conçu TEPOQAL au moyen d'un outil wiki : Tiki Wiki, une application Web de gestion de contenu et de travail collaboratif 29 .

Tiki Wiki offre de nombreux avantages par rapport à des bases de données conçues expressément pour un contenu bibliographique. Parce qu'il n'y a pas de droits d'auteur, nous ne payons aucune licence ni mise à jour et nous évitons l'éventuelle incompatibilité de la plateforme avec d'autres outils, ce qui se produit souvent avec les logiciels commerciaux. De plus, Tiki Wiki nous permet de réaliser d'importantes économies d'argent et de ressources humaines. Grâce à l'interface conviviale et intuitive de Tiki Wiki, chaque membre peut entrer des données et les mettre à jour avec un minimum de formation et de supervision. Tiki Wiki, comme tout autre wiki, permet à l'usager d'accéder au contenu et de le modifier à tout moment, depuis n'importe quel endroit, du moment qu'il est muni d'un fureteur et d'une connexion Internet.

L’application Tiki Wiki était déjà installée sur le serveur du CRTL; ce puissant moteur wiki utilise le langage de programmation PHP, MySQL comme système de gestion de bases de données et Smarty comme générateur de modèles. Comparons les détails techniques de notre projet avec ceux de la base de données CCL :

Regroupé en un ensemble sur un serveur Windows, EasyPHP (www.EasyPHP.org) comprenait déjà tous les éléments Open Source essentiels: un serveur Web Apache; $M y S Q L$, une base de données en ligne; $P H P$, un script de langage qui produit des pages Web à partir de MySQL; et le plus important, phpMyAdmin, une interface en ligne à utilisateurs multiples pour MySQL, construite à l'aide de PHP. (Taylor-Johnston, 2004, p. 181).

\footnotetext{
28 Voir http://www.linguistech.ca/.

${ }^{29}$ Pour plus d'informations, voir la page officielle du logiciel, http://info.tiki.org/tiki-index.php.
} 
L'outil que nous avons conçu est essentiellement le même que celui pensé par l'équipe de la bibliographie CCL il y a dix ans. Tout compte fait, la technologie de base (PHP et MySQL) n'a pas changé de manière radicale depuis 2001 . Toutefois, le nombre de logiciels ouverts, lui, a augmenté considérablement et leur usage s'est simplifié. La grande différence technique entre la bibliographie CCL et TEPOQAL réside dans l'interface : l'équipe de la bibliographie CCL a choisi l'application phpMyAdmin, alors que nous avons choisi Tiki Wiki. Comme il est précisé dans le Guide Tiki Wiki en ligne de Linguistech, « l’un des principaux avantages de l'application Tiki Wiki est de pouvoir revenir en arrière si une modification ne convient pas. Il est toujours possible de récupérer une version précédente d'une page éditée 30 ». Extrêmement conviviale, cette interface du site Web de Linguistech allait nous permettre de créer notre base de données sans avoir besoin d'acquérir des connaissances préalables en programmation HTML.

\section{La conception}

Une fois l'outil sélectionné, l'équipe de TEPOQAL a tenu sa première réunion avec un programmeur-conseil du CRTL, expert en Tiki Wiki. Comme il fallait s'y attendre, les défis liés à l'exécution du volet informatique d'un projet pensé par une équipe provenant du domaine des lettres ont été nombreux. Pendant que notre équipe s'attardait à la sélection et à la hiérarchisation des données à diffuser ainsi qu'aux subtilités lexicales du titre des champs, le programmeur-conseil tentait de nous ramener aux préoccupations d'ordre informatique, par exemple au caractère fonctionnel de l'outil, à sa structure et à la logique des processus informatiques, qui étaient invisibles à nos yeux, mais nécessaires à la création d'une base de données. Grâce à cet échange interdisciplinaire, nous avons appris que pour atteindre notre

\footnotetext{
30 « Guide Tiki Wiki - Historique », http://linguistech.ca/9x/Guide+Tiki+Wiki+-+Historique. La page n'existe plus aujourd'hui.
} 
objectif, l'outil de diffusion est tout aussi important que l'information à diffuser. Aussi, plus un outil à code ouvert est souple, moins il est précis et facile à personnaliser. Un logiciel à code ouvert est générique; son adaptation à chaque projet dépend de la capacité technique d'une équipe, mais aussi de sa créativité pour trouver différentes solutions pour répondre aux besoins du projet. Au bout du compte, ce n'est pas l'outil Tiki Wiki qui s'est adapté à nos besoins, mais bien nous qui avons dû nous adapter à lui.

Au cours de la première réunion, le programmeur-conseil nous a aidés à concevoir le squelette de TEPOQAL. Il a créé un formulaire (tracker) expressément pour notre base de données sur le serveur du CRTL. Il a créé les comptes, défini les paramètres d'accès et les autorisations pour chaque membre de l'équipe. Il a défini et établi avec nous l'ordre des colonnes du formulaire, et nous avons déterminé avec lui quels types de données y seraient entrés. Il nous a finalement enseigné comment créer, modifier et supprimer des fiches. Sa démonstration visait surtout à nous montrer qu'il ne fallait pas avoir peur de découvrir ni d'explorer les fonctions de Tiki Wiki. Après cette première formation, nous avons poursuivi le travail de façon autonome. Même si nous pouvions poser des questions ponctuelles au programmeur-conseil du CRTL, nos principales ressources ont été la méthode par essais et erreurs, les forums d'aide, la documentation de la communauté Tiki Wiki et la communication entre les membres de l'équipe.

La base de données TEPOQAL contient des fiches numérotées, qui correspondent chacune à un titre de la liste bibliographique. Les fiches sont créées au moyen d'un modèle contenant des champs, disposés selon un ordre préétabli et dont le type est déterminé par les données à entrer. Ci-après se trouvent les différents champs faisant partie d'une fiche, leurs caractéristiques ainsi que leur position. À des fins d'uniformisation, nous avons décidé de respecter dans la mesure du possible le protocole bibliographique MLA. Cela nous a d'ailleurs 
obligés à ajouter à chaque fiche un champ texte réservé à la référence bibliographique, car la base de données Tiki Wiki ne génère pas automatiquement de références selon un protocole préétabli (contrairement à des logiciels conçus spécifiquement à cet effet, comme EndNote).

Nom
Titre espagnol
Titre français
Auteur
Directeur
Sexe de l'auteur ou du dir ecteur
Traducteur
Sexe du traducteur
Type d'édition
Langue d'édition
$\quad$ français/espagnol/espagnol
Ville(s) d'édition
Pays d'édition
Maison(s) d'édition
Année d'édition
ISBN
Remarques
Page couverture
Référence bibliographique

\begin{tabular}{ll}
\multicolumn{1}{l}{ Type } & Options \\
champ texte & \\
champ texte & \\
champ texte & \\
champ texte & \\
liste déroulante & Masculin/Féminin \\
champ texte & \\
liste déroulante & Masculin/Féminin \\
liste déroulante & Recueil/Anthologie \\
liste déroulante & \\
& \\
avec champ de texte autre \\
champ texte \\
champ texte
\end{tabular}

Au moment de concevoir la base de données, la bibliographie statique comprenait 100 titres (recueils individuels et anthologies) et pour chacun d'entre eux, il a fallu remplir une fiche. Pour cette étape, les titres à vérifier et à entrer dans la base de données ont été répartis parmi les membres de l'équipe. L'attribution de titres parmi les assistants a fait en sorte qu'ils étaient autonomes et responsables de leurs propres fiches. Chaque assistant disposait de son propre compte dans la base de données, auquel il était possible de se connecter n'importe quand depuis n'importe quel ordinateur du moment que celui-ci était muni d'un fureteur et d'une connexion Internet. La base de données constitue donc un espace virtuel commun où les assistants peuvent consulter et modifier les fiches créées par n'importe quel utilisateur. 


\section{L'évolution}

Comme dans tout travail d'équipe, la coordination et la communication sont essentielles pour préserver l'unité et l'uniformité. L'équipe a donc décidé de créer un centre virtuel de référence et de communication pour la base de données. Conçu sur le site Wikispaces ${ }^{31}$, cet outil sert de carnet de bord, permet d'éviter les dédoublements des questions-réponses et vise à uniformiser l'entrée de données grâce à la contribution de tous les participants au projet. Grâce à Wikispaces, nous avons réussi à échanger régulièrement sur diverses questions et à prendre certaines décisions concernant l'uniformisation des données ${ }^{32}$. Le forum Wikispaces ne visait pas à remplacer la communication en personne, mais à la compléter puisqu'il permettait notamment la diffusion de guides, d'instructions et de protocoles établis lors de réunions de travail.

$\mathrm{Au}$ fur et à mesure que nous avons entré les données dans TEPOQAL, nous nous sommes familiarisés avec l'outil, et la structure originale du formulaire s'est transformée : de nouveaux champs ont été créés et d'autres ont été modifiés ou supprimés. L'exemple le plus représentatif de l'évolution est sans doute la subdivision de TEPOQAL en deux bases de données. Au début, nous avions pensé pouvoir entrer toutes les informations dans un seul outil. Cependant, en raison des difficultés techniques que représentaient la fusion et la gestion de divers types de données, nous avons décidé de créer deux outils distincts : TEPOQAL I contient les renseignements au sujet des recueils individuels et des anthologies collectives, alors que TEPOQAL II contient les données liées aux poèmes traduits contenus dans les

\footnotetext{
${ }^{31}$ Notre forum privé se trouve au tepoqal.wikispaces.com.

32 À titre d'exemple, les sujets suivants ont fait l'objet de discussions : les ISBN multiples; la langue, l'ordre et la ponctuation des données liées aux villes et aux pays; les noms des maisons d'édition et leur ordre dans le cas des coéditions; la différence entre l'auteur et le directeur de publication dans un même champ d'entrée; les autres langues d'édition; l'insertion de champs supplémentaires pour un deuxième et un troisième traducteur; la taille, la résolution et le format des images de pages couvertures; l'insertion d'un champ supplémentaire pour une deuxième page couverture dans le cas des coéditions; le partage de sources fiables pour la vérification d'information; la découverte de nouveaux titres; les questions d'ordre technique sur l'utilisation du formulaire Tiki Wiki.
} 
anthologies. Grâce à ce dédoublement, nous pouvons traiter chaque poème comme un élément distinct de l'anthologie où il figure. TEPOQAL II permet donc de faire une recherche non seulement par auteur, mais aussi par titre de poèmes (français ou espagnol) des anthologies de TEPOQAL I. À ce jour, nos recherches nous ont permis de créer dans TEPOQAL II plus de 700 fiches consultables et filtrables contenant le titre français du poème original, sa traduction espagnole, le nom du poète et de son ou ses traducteurs ainsi que les autres informations bibliographiques se rattachant à la publication. Notamment, cet outil permettra de savoir en un clic de souris quels poètes québécois sont le plus souvent inclus dans des anthologies traduites en espagnol en Amérique latine, et lesquels de leurs poèmes sont le plus souvent sélectionnés.

\section{Quelques pistes d'avenir}

Nos bases de données, dont la mise en ligne a été effectuée à l'été 2013, sont munies d'un moteur de recherche intégré. Dans TEPOQAL I, les usagers pourront effectuer des recherches par nom d'auteur et de traducteur en ordre alphabétique croissant et décroissant, par année de publication, par maison d'édition, par pays, par type de publication, etc. Une fois que le module d'extension de filtres dynamiques sera activé, les manières de filtrer les données offertes par Tiki Wiki seront multiples. Nous sommes actuellement en train d'explorer ces fonctionnalités avancées du formulaire, qui nous ont été présentées par le programmeurconseil du CRTL lors d'une nouvelle formation-éclair au sujet des filtres dynamiques et de la conception du site Web qui hébergera les bases de données à même Linguistech.

À l'origine, l'interface de consultation de TEPOQAL devait être trilingue : en français et en espagnol puisqu'il s'agit de poésie québécoise traduite vers l'espagnol, et en anglais pour que les renseignements soient accessibles dans l'autre langue officielle du Canada. En théorie, 
il est possible de rendre l'interface trilingue grâce à l'organisation du formulaire Tiki Wiki en colonnes, en tableaux et en étiquettes. Cependant, pour la première étape de familiarisation avec l'outil, nous avons décidé de travailler en français seulement. Éventuellement, si le temps ainsi que les ressources techniques et humaines le permettent, nous pourrons rendre l'interface trilingue.

Du point de vue technique, la mise à jour des bases de données est simple, car elles sont en format électronique. Nous avons prévu créer une section «Contribution » pour que les chercheurs, les auteurs, les éditeurs, les traducteurs et le grand public puissent communiquer à notre équipe de nouveaux renseignements sur les publications déjà répertoriées ou à ajouter. Selon Lane-Mercier, «à l'instar des historiens qui visent à sonder les silences de l'histoire, les traductologues ont tout à gagner à se pencher sur les silences (exclusions, omissions, lacunes, erreurs) des bases de données existantes, quelles qu'elles soient » (2014b, p.523). L'objectif du projet TEPOQAL était précisément de combler les lacunes des répertoires existants en ce qui a trait aux traductions étrangères d'œuvres québécoises et de mettre au jour un phénomène jusqu'alors peu étudié : celui de la diffusion hispano-américaine de la culture québécoise au moyen de la traduction de sa poésie. Nos deux bases de données pourront, espérons-le, rester des sources d'information fiables, exhaustives et régulièrement actualisées au sujet des traductions espagnoles de la poésie québécoise de langue française qui circulent en Amérique latine, et donner lieu à d'autres recherches du même genre.

\section{Bibliographie}

AUDET, Martine (2010), El amor de los objectos/L'amour des objets, traduction de Mercedes Roffé, New York, Pen Press.

Base de données TEPOQAL, http://linguistech.ca/tepoqal. 
BEausoleiL, Claude (2003), Gran hotel de extranjeros/Grand hôtel des étrangers, traduction de Bernardo Ruiz, Trois-Rivières et Cuauthémoc (Mexique), Les Écrits des Forges et Plan C Editores.

Beausoleil, Claude (dir.) (2008), Poesía México-Quebec Tomo I/Poésie Mexique-Québec Tome I, traduction de María Muller et de Patricia Ireta, México et Trois-Rivières, UNAM et Les Écrits des Forges.

BERNIER, Silvie (1986), «À la croisée des champs artistique et littéraire : le livre d'artiste au Québec, 1900-1980», Voix et Images, vol.11, no 3, p.528-536, http://id.erudit.org/iderudit/200586ar.

Bibliothèque et Archives Canada, Amicus, catalogue national canadien, http://amicus.collectionscanada.gc.ca/aaweb/aaloginf.htm.

Bibliothèque et Archives nationales du Québec, catalogue Iris, http://www.banq.qc.ca/accueil/.

Brossard, Nicole (2000), Vértigo del Proscenio/Vertige de l'avant-scène, traduction de Monica Mansour, Trois-Rivières et México, Les Écrits des Forges et Ediciones El Tucán de Virginia.

Cordoba SERrano, María Sierra (2007), «La fiction québécoise traduite en Espagne: une question de réseaux», Meta, vol. 52, $\mathrm{n}^{\circ} 4, \quad$ p. 763-792, http://id.erudit.org/iderudit/017696ar.

CYR, Gilles (2001), Songe que je bouge/Piensa que no estoy, traduction de Benjamín Valdivia, Querétaro, Editorial Fuera de Comercio.

Description du projet de recherche "Canada in Latin America », http://artsites.uottawa.ca/canada-latin-america/fr/research-project/.

DoRion, Hélène (2003), Orígenes/Portraits de mer, traduction de Carlos Duarte i Montserrat, New York, Pen Press.

DumonT, François (1999), La poésie québécoise, Montréal, Boréal, coll. « Boréal Express ».

Éditions du Noroît, http://test.lenoroit.com/wordpress/.

GIRARD, Hélène (1988), «Bibliographie de France Théoret », Voix et Images, vol. 14, no 1, p. 5769, http://id.erudit.org/iderudit/200753ar.

Grant, Pamela et Kathy Mezer (2007), «Establishing an Online Bibliographic Database for Canadian Literary Translation Studies », dans Yves Gambier, Miriam Shlesinger et Radegundis Stolze (dir.), Doubts and Directions in Translation Studies, Amsterdam et Philadelphie, John Benjamins, p. 73-83.

ImBERT, Patrick (1998), Antología del Noroît, traduction de Sonia Yebara, Montréal, Le Noroît.

LANE-MERCIER, Gillian (2014a), «La fiction anglo-québécoise en traduction française depuis 1990 : agents, agences et textes », Recherches sociographiques, vol. 55, no 3, p. 531-558. 
LANE-MERCIER, Gillian (2014b), «Les carences de la traduction littéraire au Canada: des bibliographies et des traditions », Meta, vol. 59, no 3, p. 517-536.

Les Écrits des Forges, Description de l'œuvre Cuerpo Salvage/Corps sauvage de Michel A. Thérien, http://www.ecritsdesforges.com/oeuvre.php?id=891.

L'infocentre littéraire des écrivains québécois, http://www.litterature.org/.

Linguistech, http://linguistech.ca/.

MAILHOT, Laurent (1997), La littérature québécoise, Montréal, TYPO.

Mailhot, Laurent et Pierre NePVeu (1990), La poésie québécoise. Des origines à nos jours, Montréal, TYPO.

MARTíneZ-ZaLCE, Graciela (2001), «Exporting Canadian Literature for Mexican Readers: the Vagaries of Translation in the Age of Globalization », Topia, n 5, p. 61-74.

MÉNDEZ, Belkis (2004), El teatro de las emociones: selección de poesía y plástica de Quebec y Cuba, Holguín, Unión de Escritores y Artistas de Cuba.

Ministère des affaires culturelles - Direction des communications (1992), La politique culturelle du Québec: notre culture, notre avenir, Québec, Gouvernement du Québec, https://www.mcc.gouv.qc.ca/fileadmin/documents/publications/politiqueculturelle1992 complet ROC.pdf.

OuELLET, François et Hans-Jürgen GREIF (2004), La littérature québécoise 1960-2000, Québec, L'instant même.

Pearltrees, https://www.pearltrees.com/\#/N-f=1 2771478\&N-fa=2771453\&N$\mathrm{p}=20231402 \& \mathrm{~N}-\mathrm{play}=0 \& \mathrm{~N}-\mathrm{s}=1 \quad 2771478 \& \mathrm{~N}-\mathrm{u}=1 \quad 278063$.

Poupaud, Sandra, Anthony Pym et Ester Torres Simón (2009), «Finding Translations. On the Use of Bibliographical Databases in Translation History », Meta, vol. 54, no 2, p. 264-278, http://id.erudit.org/iderudit/037680ar.

Pozier, Bernard (2009), Las pirámides del corazón/Les pyramides du coeur, traduction de Gabriel Martin, Tlaquepaque (Mexique) et Trois-Rivières, Mantis Editores et Les Écrits des Forges.

RIOPEL, Jean-Éric (2004), Mariposas refractadas/Papillons réfractaires, traduction d'Isabel Jazmín Ángeles, Guadalajara et Trois-Rivières, Editorial Paraíso Perdido et Les Écrits des Forges.

Rivet, Mélanie (n.d.), « Linguistech : les technologies langagières au bout des doigts », http://ti ceducation.org/papers/download/243 (n'est plus en ligne).

RoY, Françoise (2003), Le voile premier/El velo uno, Tlaquepaque (Mexique) et Trois-Rivières, Mantis Editores et Écrits des Forges. 
RoY, Françoise (2016), " "No calmes los dragones", otra obra maestra de Jean-Marc Desgent », Blanco móvil, http://www.blancomovil.com.mx/index.php/contenidos/ensayos/125-nocalmes-los-dragones-otra-obra-maestra-de-jean-marc-desgent.

SABIDO SANCHEZ, Fernando (2012), « Poetas antología universal: màs de 11100 poetas de 180 países », $\quad$ https://fernando-sabido-sanchez.blogspot.ca/2012/02/6129-emilenelligan.html.

SAPIRO, Gisèle (dir.) (2008), Translatio. Le marché de la traduction en France à l'heure de la mondialisation, Paris, CNRS Éditions.

Solis, Ricardo (2008), "Publican en español la obra completa de Garneau », La Jornada Jalisco, http://www.lajornadajalisco.com.mx/2008/01/04/index.php?section=cultura\&art

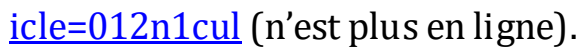

STRATFORD, Madeleine (2008), « La identidad quebequense traducida al español: difusión de la poesía quebequense en el mundo hispánico », dans Ana María Granero de Goenaga (dir.), La traducción: Hacia un encuentro de lenguas y culturas, Córdoba, Editorial Comunicarte, p. 143-166.

STRATFORD, Madeleine (2013a), “ “Voilà Québec en México!” : Le rayonnement des poètes québécois dans la revue de presse de la Foire du livre de Guadalajara de 2003 », Palabres, vol. 12, nos 1-2, p. 253-269.

STRATFORD, Madeleine (2013b), « Cuando Latinos del Norte migran hacia el Sur: la poesía de Quebec traducida en Hispanoamérica de 2000 al 2010 », Sendebar, no 24, p. 271-290, http://revistaseug.ugr.es/index.php/sendebar/article/view/652/1651.

STRATFORD, Madeleine (2013c), «Escritos de ferias y forjas: cuatro antologías de poesía de Quebec en México », Hermeneus, n 15, p. 251-290.

STRAT FORD, Madeleine (2014), « La double vie d'une même anthologie : 15 poètes du Québec au Mexique et en Argentine ", Journal of Canadian Studies/Revue d'études canadiennes, vol. 48, no 2, p. 146-168, https://doi.org/10.3138/jcs.48.2.146.

TARDIF, Dominic (2015), "Patrice Desbiens: partout et nulle part », Le Devoir, 17 octobre, http://www.ledevoir.com/lire/452696/poesie-patrice-desbiens-partout-et-nulle-part.

TAYLOR-JOHNSTON, John (2004), « Une bibliographie en ligne des Lettres : un modèle à code source libre. Le cas de www.CompCanLit.ca », Documentation et bibliothèques, vol. 50, n 2, p. 177-188, http://id.erudit.org/iderudit/1030082ar.

ThEORET, France (1981), Bloody Mary, traduction de Lazlo Moussong, Sara Diazmunoz et Brigitte Morissette, México, La bolsa y la vida ediciones.

Tiki Wiki CMS Groupware, https://tiki.org/tiki-index.php.

Toronto Public Library (2016), "Les auteurs franco-ontariens à l'honneur: Michel A. Thérien ", http://torontopubliclibrary.typepad.com/en francais/2016/08/les-auteursfranco-ontariens-\%C3\%A0-lhonneur-michel-a-.html. 
WorldCat, http://www.worldcat.org/.

\title{
Résumé
}

Depuis 1980, la poésie québécoise circule en Amérique latine, surtout au Mexique. Elle est donc en principe déjà «mondialisée». Toutefois, les versions espagnoles sont souvent difficiles d'accès : soit elles ne sont pas répertoriées, soit les références sont incomplètes ou erronées. Pour remédier à ce manque d'accessibilité, nous avons conçu deux bases de données sur Tiki Wiki, un logiciel libre de gestion de contenu et de travail collaboratif. L'une contient une bibliographie exhaustive des anthologies et recueils traduits ; l'autre, un répertoire des poèmes inclus dans les anthologies. Ces bases constituent de précieuses sources d'information pour traducteurs, traductologues et comparatistes et contribuent à une diffusion internationale tangible de la poésie québécoise traduite en espagnol en Amérique latine. Nous présenterons ici la genèse des outils, de la collecte des données à la conception et à la mise en ligne imminente. Nous traiterons des défis que nous avons relevés durant le processus et les comparerons à ceux rencontrés par les créateurs de la Bibliographie d'études comparées des littératures canadienne, québécoise et étrangère, pionniers en diffusion électronique de contenu bibliographique.

\begin{abstract}
Since 1980, Quebec poetry has been circulating in Latin America, especially in Mexico. It can thus be viewed as already "globalized". However, the Spanish versions are often hard to find: either they are not listed, or the existing references are incomplete or erroneous. In order to remedy this lack of accessibility, we have developed two databases on Tiki Wiki, an opensource application for content management and online collaboration. The first contains an exhaustive bibliography of collective anthologies and individual poetry books in Spanish translation, and the second, a comprehensive list of poems included in the anthologies. These are precious resources for translators, translation scholars and researchers in comparative literature, and they contribute to a true globalized distribution of Quebec poetry in LatinAmerican Spanish translation. This article presents how the tools were developed, from collecting the necessary data to conceiving the databases soon to be online. The main challenges of the process will be treated and compared to those met by the creators of the Bibliography of Comparative Studies in Canadian, Québec and Foreign Literatures, pioneers in electronic dissemination of bibliographical data.
\end{abstract}

